\title{
PAPÉIS DAS EMPRESAS E O CONSUMO SUSTENTÁVEL NA VISÃO DE ESPECIALISTAS BRASILEIROS
}

\author{
Verônica Macário de Oliveira \\ Doutora em Administração \\ Universidade Federal de Campina Grande \\ Campina Grande - Paraíba - Brasil \\ veronicamacario@gmail.com \\ Carla Regina Pasa Gomez \\ Doutorado em Engenharia de Produção \\ Universidade Federal de Pernambuco \\ Recife - Pernambuco - Brasil \\ carlapasagomez@gmail.com \\ Suzanne Érica Nóbrega Correia \\ Doutorado em Administração \\ Universidade Federal de Pernambuco \\ Recife - Pernambuco - Brasil \\ suzanne.enc@gmail.com
}

\section{RESUMO}

Este artigo teve como objetivo analisar os papéis das empresas para promover o consumo sustentável no contexto brasileiro. Considera-se que as empresas desempenham papéis relevantes no processo de promoção do consumo sustentável e que as suas responsabilidades não se limitam às questões tecnológicas, mas incorporam, também, ações e estratégias que se estendem nas relações com os demais stakeholders e contemplam mudanças estruturais e culturais. Assim, em termos de procedimentos metodológicos, foi realizada uma pesquisa junto a 40 especialistas brasileiros na temática, tendo como base o uso do Policy Delphi, para avaliar a relevância dos papéis identificados em amplo levantamento bibliográfico de base nacional e internacional. Os resultados demonstram que, dos 12 papéis analisados, apenas o lobby, no nível macro, não foi validado como relevante. Assim, a principal contribuição deste estudo consistiu na identificação dos papéis das empresas para promover o consumo sustentável e a sua validação junto a um conjunto de especialistas brasileiros. Porém, muitos dos papéis analisados nesse estudo necessitam ainda de um processo de institucionalização para serem implantados e disseminados entre as empresas. Além disso, atenta-se para as evidências de que as condições estruturais e contextuais de promoção do consumo sustentável dependem fortemente da articulação entre as empresas, os governos e a sociedade civil, sem os quais as possibilidades de ações isoladas por parte das empresas perdem efetividade.

Palavras-chave: Consumo sustentável; Desenvolvimento sustentável; Papéis das empresas; Responsabilidade socioambiental empresarial.

\section{BUSINESS ROLES AND SUSTAINABLE CONSUMPTION IN THE VIEW OF BRAZILIAN EXPERTS}

\section{ABSTRACT}

This paper analyzes the roles of business to promote sustainable consumption in the Brazilian context. Business plays important roles in the process of promoting sustainable consumption and responsibilities are not limited to technological issues. They also incorporate actions and strategies that extend into relations with other stakeholders and contemplate structural and cultural changes. In terms of methodological procedures, a research was carried out to 40 Brazilian specialists using the Policy Delphi to evaluate the relevance of the papers identified in a wide bibliographic survey of national and international basis. The results show that, from the 20 papers analyzed, only the lobby, at the macro level, wasn't validated as relevant. Thus, the main contribution of this study was the identification of the roles of business to promote sustainable consumption and its validation among a group of Brazilian specialists. However, many of the roles analyzed in this study still require a process of institutionalization to be implemented and disseminated. In addition, we focused on the evidence that the structural and contextual conditions for the promotion of sustainable consumption depend heavily on the articulation between companies, governments and civil society, without which the possibilities of isolated actions by the business lose effectiveness.

Key words: Business roles; Corporate social and environmental responsibility; Sustainable consumption; Sustainable development. 
Papéis das empresas e o consumo sustentável na visão de especialistas brasileiros

\section{INTRODUÇÃO}

As discussões recentes sobre desenvolvimento sustentável enfatizam que economias sustentáveis não devem focar apenas em mudanças nos processos produtivos das empresas, mas serem construídas em torno de produtos e serviços, bem como em padrões e níveis de consumo sustentáveis. Tal constatação amplia as responsabilidades socioambientais das empresas para além de suas atividades produtivas e as desafiam e pressionam para exercerem influências nas relações com seus stakeholders em ações e estratégias que possibilitem a promoção de mercados sustentáveis (Michaelis, 2003; Jackson \& Michaelis, 2003).

As empresas foram reconhecidas como fundamentais para a criação da tecnologia e riqueza que transformou a sociedade no século $\mathrm{XX}$, fornecendo a maior parte dos produtos e serviços que as pessoas consomem, o que permite pressupor que elas também são formadoras dos impactos ambientais do consumo (Jackson \& Michaelis, 2003). Desse modo, elas podem desenvolver algumas ações que contribuam com mudanças nos padrões e níveis de consumo atuais, de modo a torná-los sustentáveis. Corroborando esse posicionamento, Michaelis (2003) enfatiza que as empresas podem promover três tipos de mudanças com foco no consumo sustentável, a saber: 1) Desenvolvimento de novas tecnologias e práticas; 2) Mudanças em incentivos econômicos e legais que permeiam tanto a produção quanto o consumo; e 3) mudanças nos valores e discursos que moldam as culturas dos negócios, governos, mídia e sociedade civil.

Com base em um levantamento bibliográfico na literatura que aborda a temática, foi possível identificar estudos que examinam o papel das empresas na promoção do consumo sustentável (Michaelis, 2003; Van Nes; Cramer, 2006; Tukker et al 2008; Muster, 2011; Dubey et al., 2016). Nesses estudos, constatou-se que as responsabilidades das empresas, no que tange a insustentabilidade dos padrões e níveis atuais de consumo, não se restringem à promoção da ecoeficiência nos seus processos produtivos e produtos (Michaelis, 2003; Muster, 2011), mas incluem as possibilidades decorrentes de suas relações com os stakeholders (Tukker et al., 2008) em questões fundamentais associadas a mudanças estruturais e culturais em contextos específicos (Jackson, 2006). Isto se insere no âmbito das discussões que ensejam a participação dos grupos de interesses nos processos de decisão quanto às estratégias e ações que devem ser implantadas nas empresas para promover a produção e o consumo sustentável.

Desse modo, a realização deste estudo emergiu da percepção de que as empresas desempenham papéis relevantes no processo de promoção do consumo sustentável, atuando como protagonista, ao lado de governos e sociedade civil, no estabelecimento de estratégias e ações que sejam capazes de promover as mudanças necessárias nos contextos locais em que serão implantadas, nos níveis de atuação macro, meso e micro. O objetivo deste artigo foi, portanto, analisar os papéis das empresas para promover o consumo sustentável no contexto brasileiro. Para tanto, foi realizada uma pesquisa junto a especialistas brasileiros na temática, a partir do uso do método Policy Delphi, para avaliar a relevância dos papéis identificados.

Em termos estruturais, este artigo se organiza em cinco seções. Além desta parte introdutória, apresenta-se, na segunda, o referencial que deu sustentação teórica para este estudo; na terceira seção, são delineados os procedimentos metodológicos utilizados; os resultados obtidos são apresentados e discutidos na quarta seção; e, por fim, têm-se as considerações finais dos autores.

\section{O CONSUMO SUSTENTÁVEL E OS PAPÉIS DAS EMPRESAS}

As discussões sobre a insustentabilidade dos atuais padrões e níveis de consumo decorrem da constatação de que há uma exploração excessiva de recursos naturais pelos sistemas produtivos que não considera a finitude dos sistemas naturais, os resíduos e rejeitos gerados nesse processo excedem a capacidade de assimilação do meio ambiente, e o acesso às riquezas produzidas é injusto e desigual (Portilho, 2005; Jackson, 2006; Abramovay, 2012). No centro dessas discussões, apontase para o estilo de vida dos consumidores e os racionalismos econômicos da produção e distribuição 
como codeterminantes dos comportamentos que conduzem aos impactos ambientais do consumo (Liu, Oosterveer, Spaargaren, 2016; Shao, Taisch, Mier, 2017), de modo que a promoção do consumo sustentável se destaca como um dos principais objetivos do desenvolvimento sustentável com o propósito de alcançar eficiência energética e de recursos, acesso a serviços básicos, desenvolver infraestruturas sustentáveis e melhorar a qualidade de vida para todos (Dubey et al., 2016).

A proposta não é focar apenas na prática individual do consumidor ou em inovações tecnológicas, mas promover mudanças estruturais mais amplas que contemplem aspectos políticos, institucionais e de mercado, seguindo a lógica de responsabilidade compartilhada, de modo a criar um contexto favorável para que os padrões e níveis atuais de consumo se tornem mais sustentáveis (Consumers International; MMA; Idec, 2005; Jackson, 2006; Costa \& Teodósio, 2011; Lorek \& Spangenberg, 2014). O consumo sustentável não é apenas o consumo de produtos sustentáveis, mas envolve várias atividades nos diferentes estágios do processo envolvido na produção primária até o consumo final (Liu, Oosterveer, Spaargaren, 2016).

Assim, constata-se que a promoção do consumo sustentável possui duas dimensões: uma com ênfase na eficiência de consumo associada aos recursos que são utilizados nos processos produtivos, o que pode ser alcançada por meio de melhorias tecnológicas (Fuchs \& Lorek, 2005) e a opção dos consumidores por produtos decorrentes destes processos e a outra com foco nas mudanças macroestruturais (Fuchs \& Lorek, 2005; Jackson, 2006), as quais devem possuir maior abrangência e clareza das ações e das estratégias voltadas para promover o consumo sustentável (Pape \& Heisserer, 2011) em níveis mais amplos. No âmbito dessa discussão, deve-se atentar que mudanças incrementais de consumo tem um potencial geral muito limitado para alcançar o objetivo almejado (Lorek \& Spangenberg, 2014). A sustentabilidade do consumo, como agenda de ações de empresas, governos e sociedade civil, precisa ser avaliada continuamente, tomando uma abordagem adaptativa, equilibrada e contextualizada na elaboração de estratégias para atingir os objetivos de dimensões determinadas em qualquer definição de sustentabilidade e seus conceitos relacionados (Lim, 2017).

Outro aspecto relevante a ser considerado é que produção e consumo são duas faces da mesma moeda. O progresso contínuo em busca da sustentabilidade do consumo depende cada vez mais de construir relações nas quais as perspectivas entre produtores e consumidores estejam integradas em processos de mudanças que visem à promoção do consumo sustentável. Um fato reconhecido é que nas últimas décadas, devido ao foco de negócios em sustentabilidade, existe uma maior oferta de produtos e serviços mais eficientes e mais limpos (Blok, Long, Gaziulusoy, Ciliz, Lozano, Huisingh, 2015; Liu, Oosterveer, Spaargaren, 2016; Shao, Taisch, Mier, 2017). Entretanto, existe também uma necessidade de efetuar alterações fundamentais nos diversos modelos de negócios e nos sistemas que os suportam para superar os desafios atuais e futuros que a sustentabilidade impõe, o que remete necessariamente a ideia de gestão, já que os padrões de produção e consumo não são sustentáveis e necessitam de uma melhor orientação (Peattie, 2007). A possibilidade de atuação das empresas na promoção do consumo deve considerar os comportamentos adotados por elas (Málovics, Csigéné, Kraus, 2008).

Assim, além dos aspectos que envolvem a eficiência na utilização de recursos naturais e a inovação em processos sob o prisma da ecologia, a literatura sobre consumo sustentável aponta que as empresas estão sendo pressionadas e estimuladas pelos diversos stakeholders a contribuírem com sua a promoção, desempenhando papéis que podem ser organizados em três níveis de atuação: 1) no nível macro, as empresas podem atuar como agentes de mudança em função de sua influência econômica para disseminar melhores práticas socioambientais no mercado; 2) no nível meso, elas podem proporcionar incentivos para que seus fornecedores estejam em conformidade com os padrões socioambientais mais elevados, bem como influenciar o comportamento dos seus consumidores (Michaelis, 2003) e; 3) no nível micro, adotando práticas de gestão socioambiental que melhorem o seu desempenho em busca da sustentabilidade (Jackson \& Michaelis, 2003; Michaelis, 2003; Schrader \& Thorgersen, 2011). 
Desse modo, a Figura 1 apresenta os níveis de atuação das empresas com os seus papéis identificados na literatura de consumo sustentável pesquisada neste estudo.

\begin{tabular}{|c|c|c|}
\hline $\begin{array}{l}\text { Nível de } \\
\text { atuacão }\end{array}$ & Papéis das empresas & Principais autores \\
\hline \multirow[t]{2}{*}{ Macro } & $\begin{array}{l}\text { Disseminar de melhores práticas socioambientais no } \\
\text { mercado }\end{array}$ & $\begin{array}{l}\text { Michaelis (2003) } \\
\text { Lim (2017) }\end{array}$ \\
\hline & Lobby & Michaelis (2003) \\
\hline \multirow{5}{*}{ Meso } & Cadeia de suprimentos sustentável & $\begin{array}{l}\text { Michaelis (2003) } \\
\text { WCBSD (2008) } \\
\text { Muster (2011) } \\
\text { Lorek e Spangenberg (2014) } \\
\end{array}$ \\
\hline & Cidadania corporativa & $\begin{array}{l}\text { Michaelis (2003) } \\
\text { Muster (2011) }\end{array}$ \\
\hline & Diálogo com stakeholders & Michaelis (2003) \\
\hline & Marketing responsável & $\begin{array}{l}\text { Michaelis (2003) } \\
\text { Tukker et al. (2008) } \\
\text { Wcbsd (2008) } \\
\text { Lim (2017) }\end{array}$ \\
\hline & Rótulos e selos & $\begin{array}{l}\text { Michaelis (2003) } \\
\text { Barber (2008) } \\
\text { Schrader e Thorgersen (2011) } \\
\text { Lorek e Spangenberg (2014) }\end{array}$ \\
\hline \multirow{5}{*}{ Micro } & Ecoeficiência & $\begin{array}{l}\text { Michaelis (2003) } \\
\text { Fuchs e Lorek (2005) } \\
\text { Wcbsd (2008) } \\
\text { Lorek e Spangenberg (2014) }\end{array}$ \\
\hline & Analisar o ciclo de vida dos produtos & $\begin{array}{l}\text { Barber (2008) } \\
\text { Fuchs; Lorek (2005) } \\
\text { Tukker et al. (2008) } \\
\text { Liu, Oosterveer e Spaargaren, } 2016\end{array}$ \\
\hline & Produtos sustentáveis & $\begin{array}{l}\text { Tukker et al. (2008) } \\
\text { Liu, Oosterveer e Spaargaren } \\
\text { (2016) } \\
\text { Shao, Taisch, \& Mier (2017) }\end{array}$ \\
\hline & Embalagens ecológicas & Barber (2008) \\
\hline & Ecotaxas & $\begin{array}{l}\text { Michaelis (2003); } \\
\text { Tukker et al. (2008) }\end{array}$ \\
\hline
\end{tabular}

Figura 1. Papéis das empresas para promover o consumo sustentável

Fonte: Elaborado pelos autores.

As empresas podem assumir a liderança na adoção de novos indicadores de sucesso baseados nos princípios de sustentabilidade. Elas podem inovar em suas formas de atuação e buscar mecanismos que possibilitem a disseminação de práticas sustentáveis. (Oliveira, Correia, \& Gomez, 2016). Uma das possibilidades envolve a adoção do princípio de valor compartilhado, o qual propõe a criação de valor econômico ao mesmo tempo em que se busca criar valor para a sociedade, abordando suas necessidades e desafios, além de promover o progresso social (Porter \& Kramer, 2011).

Elas podem atuar em nível macro como agentes de mudança política em função de influência econômica que exercem no mercado para disseminar melhores práticas socioambientais e realizar lobby como forma de influenciar as práticas governamentais. Tais atores também podem atuar no nível meso e tomar a iniciativa de tentar novas estruturas com incentivos para melhorar seu desempenho ambiental e proporcionar incentivos para que seus fornecedores estejam em conformidade com padrões socioambientais mais elevados, bem como buscar influenciar seus consumidores (Michaelis, 2003). E, no nível micro, as empresas devem incluir preocupações ambientais nos seus modelos de negócios, adotando processos de ecoeficiência e ecoinovação, que 
buscam reduzir os impactos ecológicos e a intensidade de uso de recursos naturais durante todo o ciclo de vida de seus produtos (WBCSD, 1997). Considera-se que grandes mudanças técnicas, em geral, envolvem alguma alteração no produto ou serviço recebido pelo consumidor e, portanto, resultam em mudanças nos padrões de consumo (Michaelis, 2003; Shao, Taisch, Mier, 2017).

A gestão da cadeia de suprimentos focaliza questões relacionadas à integração de processos entre os elos da cadeia (Pires, 2009). Entretanto, diante da elevação da discussão sobre questões socioambientais associados aos questionamentos sobre os impactos da produção e do consumo, emergiram novos interesses despontando a ideia de cadeia de suprimentos sustentável (Brito \& Berardi, 2010), com foco na responsabilidade socioambiental compartilhada (Tukker et al., 2008). Isso requer um conjunto de mudanças gerenciais, estruturais e organizacionais para que se dissemine nas relações entre os elos da cadeia a preocupação com os impactos socioambientais que as suas atividades provocam, de modo que se busquem formas de evitá-los ou mitigá-los, contribuindo para uma maior abrangência das ações que visam o consumo sustentável. Ocorre a disseminação de práticas sustentáveis em todos os elos da cadeia de suprimentos para que as ações de cada intermediário se tornem responsáveis (Michaelis, 2003; Muster, 2011). Para tanto, é necessário promover o fluxo de informação padronizada ao longo da cadeia sobre os aspectos ecológicos, bem como sobre as normas sociais que devem ser seguidas pelas empresas (Lorek \& Spangenberg, 2014; Shao, Taisch, Mier, 2017). Outro fator associado à promoção da cadeia de suprimentos sustentável é a adoção da logística reversa como uma estratégia operacional que inclui processos de reuso, reciclagem e descarte de produtos depois de sua vida útil (Bowersox, Closs, Cooper, Bowersox, 2014).

A cidadania corporativa envolve um alto padrão de conduta ética das empresas para com seus stakeholders (Michaelis, 2003) que são definidos como qualquer organização ou quaisquer indivíduos legitimamente interessados nas ações da empresa, tais como empregados, acionistas, governos, organizações-não-governamentais (ONGs), comunidades afetadas, fornecedores e clientes. Carroll (1998) destaca que a cidadania corporativa ocorre a partir da incorporação de práticas responsáveis das empresas nas dimensões econômica, legal, ética e filantrópica. Assim, o autor define que o bom cidadão corporativo atende aos seus interesses econômicos, cumpre as leis, possui comportamento ético e exerce filantropia com a comunidade e seus demais stakeholders. Fuchs e Lorek (2005) destacam que a busca por ações mais concretas para promover o consumo sustentável se baseiam na esperança do aumento na cidadania corporativa.

O diálogo com os stakeholders se relaciona ao processo de interação entre a empresa e suas partes interessadas no processo de tomada de decisão (Michaelis, 2003; Monte \& Peplys, 2008; Silva \& Cândido, 2014). As empresas precisam desenvolver culturas que sejam congruentes com os valores de seus funcionários, clientes, investidores, e as comunidades nas quais elas operam, engajando-se em diálogos contínuos para estabelecer e rever um entendimento compartilhado de valores, metas e prioridades (Michaelis, 2003), com foco no entendimento mais amplo das mudanças necessárias para minimizar os impactos ambientais das atividades de produção e consumo. Daí a necessidade de as empresas adotarem mecanismos de interação com os stakeholders para levar em consideração os seus anseios nas suas decisões e ações, em um processo de comunicação que proporcione legitimidade na sociedade. Assim, como partes interessadas, eles podem legitimar as empresas como socialmente responsáveis, de acordo com a forma como elas se comportam diante das questões socioambientais.

O desenvolvimento das estratégias de marketing deve ser combinado com um forte senso de responsabilidade socioambiental. O marketing responsável emerge como um novo mecanismo de comunicação das novas práticas organizacionais alinhadas aos valores empresariais que visam estimular uma mudança cultural coletiva, considerando a influência que o marketing e a publicidade podem ter sobre os consumidores e a sociedade (Michaelis, 2003; Tukker et al., 2008). Essas abordagens para promover o consumo sustentável são, muitas vezes, baseadas em técnicas de marketing social que são "orientadas para o cliente" e usam conceitos e ferramentas de empresas de marketing e publicidade comercial. A pesquisa, o planejamento, a segmentação, o uso de diferentes 
ferramentas e a manutenção do esquema durante um período até que os resultados sejam alcançados são os principais ingredientes para que as ações de marketing responsável sejam bem-sucedidas (OECD, 2008).

Disponibilizar informações sobre os produtos por meio de rótulos e selos é uma forma de informar os consumidores sobre os aspectos ambientais dos produtos, de modo a auxiliá-los nas suas decisões de consumo (Shao, Taisch, Mier, 2017). Pode-se utilizar rotulagens do tipo autodeclaração para informar sobre as características benéficas ao meio ambiente presentes em produtos ou serviços específicos, tais como biodegradabilidade, retornabilidade, uso de material reciclado, eficiência energética e outras (Barbieri, 2007). Também são usados para fornecer incentivos aos consumidores para um maior envolvimento na coleta e reciclagem de produtos e resíduos de embalagens (OECD, 2008). No entanto, as deficiências gerais dos rótulos, incluindo baixos níveis de consciência do consumidor, as diferenças de critérios entre produtos, a concorrência no mercado entre vários tipos de rotulagem, bem como a proliferação de rótulos voluntários no mercado levam à confusão do consumidor entre autodeclarações e rótulos certificados por terceiros (OECD, 2008).

Considera-se que $30 \%$ a $80 \%$ dos impactos ambientais de um produto são decididos na sua fase de concepção. Daí a importância de adotar o princípio de ecoeficiência e a avaliação do ciclo de vida (Clark, 2007) que buscam conciliar crescimento econômico e mitigação dos impactos no meio ambiente (Fuchs \& Lorek 2005). As preocupações não se limitam ao volume de produção e aos tipos de bens e serviços produzidos, mas também incluem os processos de produção, os recursos naturais extraídos como fontes de matéria-prima, bem como os resíduos e a poluição resultantes dos processos produtivos (Barber, 2008; Brizga, Mishchuk, Golubovska-Onisimova, 2014). Melhorias na ecoeficiência do consumo significam uma redução no consumo de recursos por unidade de consumo, como água, energia e outros recursos inerentes aos produtos, devido a melhorias nos processos de produção (Fuchs \& Lorek, 2005).

Estas questões poderiam influenciar diretamente no desenvolvimento de produtos sustentáveis que minimizem a utilização de recursos e o impacto ambiental que estes possam gerar. Desse modo, os avanços localizados e setoriais da ecoeficiência são evidentes, porém um dos seus maiores obstáculos está nas oportunidades de ganho econômico oferecidas pelas práticas baseadas em métodos predatórios, o que inibe a disseminação de inovações que poderiam economizar materiais e energias, mas com acréscimos de custos, em decorrência da incapacidade de se obter consenso em eliminar os métodos convencionais de produção e da dificuldade de se obter regulação estatal (Abramovay, 2012). Ademais, o uso de embalagens ecológicas (Tukker, et al., 2008; Barber, 2008) pode diminuir a utilização de recursos naturais e promover um menor impacto ambiental.

As respostas das empresas aos incentivos e regulamentações do governo deveriam considerar a influência que este exerce sobre as suas práticas. Impostos e taxas podem ser efetivos para influenciar o comportamento dos consumidores em direção à sustentabilidade ao elevar os preços de produtos menos sustentáveis. Essas ferramentas ajudam a internalizar as externalidades negativas e permitir que o mercado desempenhe o papel crítico de mudar padrões de compras. Os impostos e encargos podem ser mais rentáveis que os regulamentos, que podem exigir esforços intensivos de monitoramento, em termos de execução e controle, e oferecem maior flexibilidade às famílias e às empresas para se adaptarem (Oecd, 2008). Geralmente as empresas se tornam passivas nessa interação, seja na obtenção de incentivos ou no atendimento às regulamentações. Uma forma de a empresa responder à atuação regulamentar do governo seria a partir da adoção de ecotaxas para internalizar as externalidades ambientais, partilhando os custos ambientais e os incentivos para a mudança em toda a cadeia de valor (Michaelis, 2003).

Os papéis apresentados possibilitam o reconhecimento das empresas como um dos principais agentes de mudança para promover o consumo sustentável. Entretanto, deve-se considerar que essa atuação não consiste em gesto unilateral do setor privado, mas é a resposta a um conjunto mais ou menos difuso de pressões que ampliam os riscos empresariais e obrigam as 
companhias a transformar os vínculos com base nos quais realizam seus negócios (Abramovay, 2012). Há toda uma complexidade na adoção de práticas socioambientais nas empresas e isto implica em grandes esforços os quais nem sempre são lucrativos.

\section{PROCEDIMENTOS METODOLÓGICOS}

O objetivo desta pesquisa foi analisar os papéis das empresas que visam promover o consumo sustentável no contexto brasileiro. Para tanto, realizou-se um estudo exploratório com o propósito de gerar novas ideias sobre a atuação das empresas no processo de mudanças que contribuam para alcançar a sustentabilidade nas atividades de consumo. Inicialmente, fez-se a identificação dos papéis das empresas na promoção do consumo sustentável, sintetizados na Figura 1 , apresentada anteriormente. Isso ocorreu com base em levantamento de amplo referencial teórico de bases nacionais e internacionais que proporcionaram a constituição, a priori, de parâmetros associados ao processo de promoção do consumo sustentável, a partir de abordagens que considerem a complexidade e abrangência das estratégias e ações a serem adotadas, de modo que os papeis analisados foram classificados em três níveis de atuação, a saber: macro, meso e micro. $\mathrm{Na}$ sequência, esses papéis foram submetidos à apreciação de especialistas.

A técnica adotada foi o Policy Delphi, baseando-se no uso estruturado do conhecimento, da experiência e da criatividade de um painel de especialistas. Pressupõe-se que o julgamento coletivo, quando organizado adequadamente, é melhor que a opinião individualizada. Esta técnica é adequada quando se pretende explorar um tópico complexo, com pouco contexto histórico e que exige parecer de especialistas para compreender as questões subjacentes (Rayens \& Hahn, 2000; Franklin \& Hart, 2007), como é o caso do consumo sustentável. A finalidade é garantir que todas as opções possíveis sejam consideradas, estimando o impacto e a consequência de qualquer ação particular, além de examinar e avaliar a aceitabilidade de qualquer opção específica (Turoff, 1997).

A amostra investigada foi não-probabilística intencional, composta por um conjunto de especialistas na temática, o que incluiu: 1) técnicos e pesquisadores que atuam em órgãos e entidades públicas na área de meio ambiente e de proteção ao consumidor, como a equipe técnica do Departamento de Produção e Consumo Sustentáveis (DPCS) do Ministério do Meio Ambiente (MMA) e gestores de Procons Municipais que apresentassem uma atuação de destaque nacional na defesa dos interesses dos consumidores; 2) acadêmicos envolvidos com pesquisas ligadas ao tema em estudo, identificados a partir de levantamento das publicações sobre a temática "consumo e sustentabilidade" Portal Periódicos, utilizando como critério a publicação de, no mínimo, dois artigos em periódico de extrato superior Qualis que abordassem o tema nos últimos cinco anos; 3) profissionais com experiências em empresas que promovem ações voltadas para promoção do consumo sustentável, selecionados na rede Linkedin por meio de uma busca contendo os termos "experiência profissional em produção e consumo sustentável"; 4) e pessoas atuantes em ONGs, responsáveis pelos empreendimentos sociais voltados para a promoção de consumo sustentável no Brasil e de defesa dos direitos dos consumidores, identificados por Rattis Teixeira (2011) e Instituto Kairós (2011). A amostra totalizou 40 especialistas investigados, conforme Tabela 1.

Tabela 1: Quantidade x Participação de especialistas no Policy Delphi

\begin{tabular}{l|c|c}
\hline \multicolumn{1}{c}{$\begin{array}{c}\text { Grupo de } \\
\text { Especialistas }\end{array}$} & $\begin{array}{c}\text { Quantidade } \\
\text { Convidada }\end{array}$ & $\begin{array}{c}\text { Quantidade de } \\
\text { Participantes }\end{array}$ \\
\hline Academia (G1) & 67 & 16 \\
Governo (G2) & 18 & 08 \\
Empresas (G3) & 13 & 08 \\
ONG's (G4) & 37 & 08 \\
Total & 135 & 40 \\
\hline
\end{tabular}

Fonte: Dados da Pesquisa. 
A primeira rodada ocorreu com a aplicação de questionários semiestruturados, disponíveis em plataforma online (GoogleDocs). Para cada papel analisado, os especialistas expressaram suas opiniões, assinalando uma escala do tipo Likert, em cinco pontos distribuídos de acordo com os níveis de relevância (5 - criticamente importante; 1 - não tem importância). Havia um espaço para comentários adicionais. Após o retorno dos questionários, na primeira rodada, foram feitos cálculos estatísticos da medida central (média $(\mu)$ ) e da medida de dispersão (coeficiente de variação $(C V)$ ) para cada variável, segundo cada grupo de especialistas e o grupo total, com o propósito de verificar os níveis de convergência e divergência nas respostas dos especialistas, bem como a reunião dos diversos comentários adicionais.

$\mathrm{Na}$ segunda rodada, os especialistas receberam um novo questionário com o sumário dos resultados obtidos na primeira rodada e o comparativo com as suas respostas, solicitando-lhes se posicionar novamente em relação às questões apresentadas. Como, nesta etapa, os questionários possuíam características individuais específicas para cada especialista, eles foram desenvolvidos em arquivo do tipo *.doc e enviados por email.

Para determinar quais os papéis que foram considerados relevantes, comparou-se a média da pontuação de todos os fatores investigados usando um ponto de corte da média de 3,8 . Isto porque foram consideradas as médias das respostas próximas ao valor 4 (muito importante) até o 5 (criticamente importante). Para identificar se haviam papéis que apresentavam uma distribuição de opiniões controversas, foram avaliados aqueles que apresentaram média inferior a 3,8 e que possuíam um alto coeficiente de variação $(\mathrm{CV})$, convencionalmente atribuído ao valor acima de $40 \%$ no resultado geral.

A aplicação da técnica Policy Delphi foi encerrada na segunda rodada, considerando que os resultados obtidos nesta etapa confirmaram o nível de relevância atribuído pelos especialistas investigados na primeira rodada. Os resultados obtidos são apresentados e discutidos na seção a seguir.

\section{RESULTADOS}

Nesta seção, analisam-se os resultados sobre os papéis das empresas no processo de promoção do consumo sustentável no contexto brasileiro, nos níveis de atuação macro, meso e micro, considerando as respostas obtidas nas duas etapas do Policy Delphi.

\subsection{PAPÉIS DAS EMPRESAS NO NÍVEL MACRO}

No nível macro foram avaliados dois papéis de atuação das empresas (Tabela 2). O primeiro papel relaciona-se à influência econômica que as empresas exercem no mercado, podendo realizar lobby com o objetivo de promover mudanças políticas ao influenciar as práticas governamentais para promover o consumo sustentável. As médias gerais obtidas nas duas rodadas foram 3,79 e 3,77. Entretanto, as medidas de dispersão demonstram que há maior heterogeneidade das respostas nos grupos de especialistas de governo (G2) e de ONGs (G4) cujas médias das respostas apontam para não relevância deste papel, enquanto os especialistas da academia (G1) e das empresas (G3) são mais coesos em suas respostas, apresentando menores medidas de dispersão e médias que expressam a relevância deste papel para promoção do consumo sustentável no contexto brasileiro.

Tabela 2: Comparativo entre os resultados das rodadas sobre os papéis das empresas no nível macro

\begin{tabular}{|c|c|c|c|c|c|}
\hline \multirow[b]{2}{*}{ Papéis de empresas (Macro) } & \multirow{2}{*}{$\begin{array}{c}\text { Grupos de } \\
\text { especialistas }\end{array}$} & \multicolumn{2}{|c|}{$\mathbf{1}^{\mathrm{a}}$ Rodada } & \multicolumn{2}{|c|}{$2^{a}$ Rodada } \\
\hline & & $\mu$ & CV & $\mu$ & $\mathbf{C V}$ \\
\hline \multirow{5}{*}{ Lobby } & Geral & 3,79 & 0,29 & 3,77 & 0,29 \\
\hline & G1 & 4,00 & 0,22 & 3,82 & 0,23 \\
\hline & G2 & 3,14 & 0,43 & 3,29 & 0,42 \\
\hline & G3 & 4,57 & 0,12 & 4,57 & 0,12 \\
\hline & G4 & 3,25 & 0,39 & 3,38 & 0,39 \\
\hline
\end{tabular}

Revista de Gestão Social e Ambiental - RGSA, São Paulo, v. 12, n. 2, p. 55-70, maio/ago. 2018. 


\begin{tabular}{c|l|c|c|c|c}
\hline \multirow{4}{*}{$\begin{array}{c}\text { Disseminar melhores práticas } \\
\text { socioambientais }\end{array}$} & Geral & 4,41 & 0,15 & 4,40 & 0,15 \\
\cline { 2 - 6 } & G1 & 4,41 & 0,12 & 4,41 & 0,11 \\
\cline { 2 - 6 } & G2 & 4,29 & 0,18 & 4,29 & 0,18 \\
\cline { 2 - 6 } & G3 & 4,71 & 0,10 & 4,71 & 0,10 \\
\cline { 2 - 6 } & G4 & 4,25 & 0,24 & 4,19 & 0,24 \\
\hline
\end{tabular}

Fonte: Pesquisa com especialistas

O lobby é considerado como um processo de interação entre empresas-governos (Michaelis, 2003), no qual as empresas são proativas e têm a possibilidade de pressionar as práticas de governo em determinadas questões. Os resultados que demonstram a divergência entre os grupos de especialistas investigados sobre a relevância do papel supracitado são ressaltados nos comentários adicionais, no quais se observam algumas ressalvas e o ceticismo daqueles quanto ao desempenho efetivo da realização de lobby por parte das empresas em prol de mudanças estruturais que visem promover a sustentabilidade, principalmente porque, em alguns casos, isto pode ir de encontro aos seus interesses financeiros.

É importante, só precisa tomar cuidado para que não ocorra o chamado greenwash, e que a pressão seja para privilegiar grupos financeiros em detrimento de outros menos poderosos" (Acad16);

"Acho perigoso colocar as empresas neste papel, pois, em última instância, seu objetivo é o lucro" (ONG7).

Assim, a principal crítica relacionada ao lobby envolve a negociação de interesses difusos a partir da influência exercida pelas empresas nas práticas governamentais. O outro papel das empresas avaliado, no nível macro, foi disseminar melhores práticas socioambientais no mercado. Os dados mostram que os especialistas consideram este papel "muito importante", com média geral de 4,41 e 4,40 nas duas rodadas e $15 \%$ de dispersão das respostas em torno da média. Segundo Michaelis (2003), alguns líderes empresariais veem o mundo corporativo como a melhor alternativa para o alcance da sustentabilidade ambiental. Considera-se que, em um mundo globalizado, as empresas são as instituições mais aptas a fornecerem as novas tecnologias e os instrumentos necessários para alcançar melhorias radicais de eficiência na utilização de recursos naturais e redução da poluição. Tukker et al. (2008) afirmam que as empresas estão, provavelmente, melhores colocadas para enfrentar os desafios da sustentabilidade por meio da inovação radical em produtos e serviços e novos modelos negócios relacionados.

Na prática, considera-se que a regulamentação do governo tem sido, muitas vezes, o fator mais importante que determina a adoção de tecnologias de controle de poluição das empresas, enquanto a melhoria na eficiência dos recursos e mudanças para tecnologias inerentemente mais limpas são mais propensas a serem motivadas pelas forças do mercado (Michaelis, 2003; Tukker et al., 2008). Os comentários a seguir corroboram neste sentido.

É fundamental que as empresas comuniquem seus resultados para que, por meio da própria concorrência, as melhores práticas sejam adotadas no maior número de casos" (EMP4);

"Importante o trabalho com inovação na linha produtiva com foco na sustentabilidade dos processos" (ONG8).

Por outro lado, o especialista (ONG2) enfatizou que "[...] já temos várias ferramentas desse tipo e são importantes, mas não ao ponto de causar mudanças sistêmicas [...]" e o especialista (ONG4) declarou que as empresas "[...] podem fazer isso sendo mediadas pelo Estado". Esses posicionamentos reforçam a necessidade de articulação entre governos e empresas na criação das condições macroestruturais que favoreçam à promoção do consumo sustentável. 


\subsection{PAPÉIS DAS EMPRESAS NO NÍVEL MESO}

No nível meso foram avaliados cinco papéis das empresas (Tabela 3). O primeiro papel avaliado foi promover a cadeia de suprimentos sustentável com foco na responsabilidade socioambiental compartilhada que está associada à indagação sobre até onde essas responsabilidades se estendem entre os elos que formam a cadeia da atividade principal da empresa e as atividades de consumo e pós-consumo (Michaelis, 2003; Muster, 2011). As médias gerais obtidas foram de 4,49 e 4,51 e os coeficientes de variação, de 16\%, nas duas rodadas. Assim, a classificação final deste papel foi "criticamente importante".

Tabela 3: Comparativo entre os resultados das rodadas sobre os papéis das empresas no nível meso

\begin{tabular}{|c|c|c|c|c|c|}
\hline \multirow[b]{2}{*}{ Papéis de empresas (Meso) } & \multirow{2}{*}{$\begin{array}{c}\text { Grupos de } \\
\text { especialistas }\end{array}$} & \multicolumn{2}{|c|}{$\mathbf{1}^{\mathrm{a}}$ Rodada } & \multicolumn{2}{|c|}{$2^{\text {a }}$ Rodada } \\
\hline & & $\mu$ & $\mathbf{C V}$ & $\mu$ & $\mathbf{C V}$ \\
\hline \multirow{5}{*}{ Cadeia de suprimentos sustentável } & Geral & 4,49 & 0,16 & 4,51 & 0,16 \\
\hline & G1 & 4,53 & 0,11 & 4,53 & 0,11 \\
\hline & G2 & 4,00 & 0,25 & 4,14 & 0,26 \\
\hline & G3 & 5,00 & 0,00 & 5,00 & 0,00 \\
\hline & G4 & 4,38 & 0,21 & 4,38 & 0,21 \\
\hline \multirow{5}{*}{ Cidadania corporativa } & Geral & 4,49 & 0,19 & 4,46 & 0,19 \\
\hline & G1 & 4,65 & 0,13 & 4,65 & 0,13 \\
\hline & G2 & 3,71 & 0,34 & 3,71 & 0,34 \\
\hline & G3 & 4,86 & 0,08 & 4,86 & 0,08 \\
\hline & G4 & 4,50 & 0,21 & 4,38 & 0,21 \\
\hline \multirow{5}{*}{ Diálogo com stakeholders } & Geral & 4,36 & 0,19 & 4,32 & 0,19 \\
\hline & G1 & 4,59 & 0,11 & 4,53 & 0,11 \\
\hline & G2 & 3,29 & 0,34 & 3,29 & 0,34 \\
\hline & G3 & 4,86 & 0,08 & 4,86 & 0,08 \\
\hline & G4 & 4,38 & 0,17 & 4,31 & 0,16 \\
\hline \multirow{5}{*}{ Marketing responsável } & Geral & 4,21 & 0,26 & 4,19 & 0,25 \\
\hline & G1 & 4,18 & 0,23 & 4,18 & 0,21 \\
\hline & G2 & 4,14 & 0,29 & 4,14 & 0,29 \\
\hline & G3 & 4,71 & 0,10 & 4,71 & 0,10 \\
\hline & G4 & 3,88 & 0,40 & 3,81 & 0,40 \\
\hline \multirow{5}{*}{ Rótulos e selos } & Geral & 4,85 & 0,08 & 4,87 & 0,07 \\
\hline & G1 & 4,94 & 0,05 & 4,94 & 0,05 \\
\hline & G2 & 4,71 & 0,10 & 4,86 & 0,08 \\
\hline & G3 & 4,71 & 0,10 & 4,71 & 0,10 \\
\hline & G4 & 4,88 & 0,07 & 4,88 & 0,07 \\
\hline
\end{tabular}

Fonte: Pesquisa com especialistas.

Para Tukker et al. (2008), as empresas são capazes de promover valores de sustentabilidade na sua cadeia de suprimentos, o que poderá favorecer a promoção da relação entre produção e consumo sustentáveis. Os especialistas acrescentaram nos comentários que esse papel pode ser operacionalizado por meio da adoção de critérios de sustentabilidade e métricas para acompanhar a sua implementação (Acad16) e que a atuação, de maneira compartilhada e integrada de ponta a ponta na cadeia de valor, é fundamental para viabilizar tanto a produção quanto o consumo sustentável (EMP4). Deve-se atentar também para os aspectos de qualificação e avaliação de fornecedores da cadeia, pontuado por outro especialista, quando enfatiza que

Qualificar e avaliar fornecedores, permite a escolha de produtos que atendam aos requisitos de sustentabilidade definidos nas premissas da empresa e faz com que aqueles que não estão adequados busquem se adequar para ganhar novos mercados (EMP7). 
Esses resultados reforçam o argumento de Michaelis (2003) de que as empresas devem procurar envolver outros intermediários na cadeia de suprimentos em uma perspectiva mais responsável que favoreça a emergência de estruturas que deem sustentação a práticas de produção e consumo sustentáveis.

Entretanto, alguns deles vinculam o efetivo desempenho desse papel às questões de regulamentação do governo e à pressão da sociedade civil, demonstrando que há uma desconfiança quanto à voluntariedade das empresas em promover esse tipo de estratégia.

A responsabilidade compartilhada é fundamental para que ocorra uma mudança nas formas de conduzir a produção de bens e serviços. Mas não acredito que ocorrerá de forma espontânea. Para que haja, de fato, um comprometimento do setor privado, é necessário o papel regulatório do Estado e a consciência (e pressão) dos consumidores" (EMP2);

Mais uma vez, avalio que a cadeia de suprimentos sustentável só existe, na iniciativa privada, como meio para reduzir custos. Se tal sustentabilidade vier sem aumento de lucro, ela não se concretiza (GOV1).

Porém, a OECD (2008) alerta para as dificuldades de execução e controle de mecanismos de regulamentação do governo que exigem esforços intensivos de monitoramento para garantir o seu cumprimento.

Quanto ao exercício da cidadania corporativa, os dados apresentados apontam que os especialistas classificam esse papel como "muito importante", com médias geral de 4,49 e 4,46 e coeficiente de variação de 19\%. Segundo Carroll (1998), a empresa cidadã exemplar se esforça para ampliar seus lucros (responsabilidade para si mesmo), ao cumprir suas obrigações de cidadania para os outros (lei, ética, e filantropia). Porém, o autor ressalta que estas responsabilidades não devem ser preenchidas sequencialmente, mas ao mesmo tempo, na busca por um modelo de cidadania corporativa que se estende nas relações com os seus diversos grupos interessados. Nesse sentido, um dos especialistas apontou para a necessidade de adoção de projetos que promovam geração de valor compartilhado. Isso corrobora Crane, Matten e Moon (2010) ao destacar que esse comportamento pode ser entendido como a relação entre empresas e sociedade ao contribuir para o aspecto da responsabilidade ambiental assumida por elas, bem como para enfrentar os atuais desafios socioambientais apontados Porter e Kramer (2011).

No que se refere ao diálogo com os stakeholders, os dados mostram que os especialistas investigados apresentaram as médias gerais de 4,36 e 4,32, classificando-o como "muito importante" e o coeficiente de variação foi de $19 \%$ em ambas as rodadas. Mais uma vez o grupo de especialistas de governo (G2) apresentou as menores médias de avaliação da relevância deste papel e os maiores coeficientes de variação, demonstrando uma maior dispersão das respostas em torno da média. A relevância deste papel é confirmada no seguinte comentário adicional:

\footnotetext{
A melhoria de processos, a diminuição do uso de recursos naturais e de consumos necessários para as atividades produtivas virão de uma constante busca da empresa por novas tecnologias, da apresentação de oportunidades disponibilizada por fornecedores e consultores, bem como do feedback dos funcionários e clientes, por isso estabelecer um canal de comunicação fácil e eficiente que permitirá que a empresa nunca esteja longe das tendências da sustentabilidade (EMP7).
}

Estas falas corroboram o argumento de Oliveira (2013) que as sociedades nas quais os stakeholders têm maior poder de organização e força política, elas tendem possivelmente a ter um poder de diálogo maior com as empresas. Isso exige que as empresas aperfeiçoem algumas ferramentas para promover esse diálogo, dentre as quais se destacam: as técnicas de gestão de conflitos e as pesquisas de opinião que buscam conhecer o que eles pensam. Por outro lado, alguns especialistas demonstraram ceticismo quanto ao desempenho desse papel por parte das empresas: 
A preocupação das empresas deve estar calcada na legislação. Responsabilidade social é gasto, não investimento. As práticas de consumo, em geral não estão alinhadas a ações de responsabilidade social por parte das empresas. O retorno não é certo..." (ACAD12);

"Esse comprometimento só acontece quando há vantagem competitiva (incentivos, imagem, adesão dos consumidores) ou riscos aos negócios caso sejam menosprezados" (EMP2).

O papel relacionado à prática do marketing responsável como um novo mecanismo de comunicação que visa estimular uma mudança cultural coletiva alinhada a um forte senso de responsabilidade socioambiental foi avaliado pelos especialistas investigados como "muito importante", com médias geral de 4,21 e 4,19 e dispersão de $26 \%$ e $25 \%$ das respostas em torno da média, nas duas rodadas respectivamente (Tabela 3). O grupo de especialistas ligados às ONGs (G4) apresentou as menores média de avaliação deste papel e o maior coeficiente de variação (40\%) nas duas rodadas, inserido no limite do critério da homogeneidade das respostas adotado neste estudo. Isso pode ter ocorrido devido à desconfiança que as ONGs, geralmente, mantêm quanto às questões que envolvem as ações de marketing das empresas e as relações socioambientais que estão inseridas nas suas práticas. Além disso, grande parte das ações de marketing das empresas sempre busca aumentar os níveis de consumo (OECD, 2008), sem refletir sobre os impactos que podem gerar na sociedade e no meio ambiente.

Porém, para contribuir com a promoção da sustentabilidade, é importante que os esforços de comunicação das empresas busquem influenciar mudanças de atitudes dos consumidores diante das questões socioambientais, considerando o impacto que as ações de marketing exercem na sociedade, conforme afirma Oliveira (2013). Nesse contexto, os comentários adicionais atentam para as dificuldades que envolvem o desempenho dessa atividade nas empresas, se coadunando com os argumentos teóricos expostos de que ela está associada à busca pelo aumento na demanda dos produtos da empresa e, consequentemente, o aumento no consumo.

O ideal é que sim, mas o marketing é desenvolvido, na prática, justamente para fazer com que o consumo seja maior (ACAD12);

As tendências de sustentabilidade devem ser uma estratégia de comunicação ao público, cada vez mais ligado aos cuidados que as empresas adotam em suas atividades produtivas e a associação da sua imagem aos critérios de sustentabilidade (EMP7);

Acho que marketing e publicidade atuam num nível muito superficial quando se pensa numa mudança profunda e significativa. O marketing e a publicidade são alienantes. Precisamos de pessoas com senso crítico e capacidade de ter suas próprias ideias e serem criativas, só a publicidade não consegue fazer isso. Nossa sociedade tem que consumir menos e eu duvido que o marketing das empresas difunda essa ideia" (ONG7).

A falta de informação sobre aspectos socioambientais dos produtos é considerada como uma barreira para a efetivação do consumo sustentável. Assim, os especialistas avaliaram como "criticamente importante" as empresas informarem aos consumidores sobre os aspectos ambientais dos produtos pelo uso de rótulos e selos, conforme os dados da Tabela 3. Há uniformidade nas respostas apresentadas pelos grupos investigados, o que pode ser observado pelos baixos coeficientes de variação obtidos.

Os consumidores, teoricamente, com base na disponibilização dessas informações, podem fazer escolhas de consumo mais conscientes e sustentáveis. Assim, os especialistas ressaltaram, nos comentários adicionais, a importância desse papel como forma mais prática de as empresas auxiliarem os consumidores nas suas decisões de consumo, além de agregar diferencial competitivo aos seus produtos.

É a base do princípio da informação do direito do consumidor. É por meio de rótulos e selos que o consumidor tem ampliado o seu poder de escolha partindo das informações prestadas pelo fornecedor (ACAD16); 
Independentemente do que pede os órgãos reguladores e normatizadores para informativos das embalagens, as empresas devem trabalhar para informar todos os critérios de sustentabilidade adotados, como um diferencial do seu produto (EMP7);

Parece ser esse o caminho mais prático, pois é impraticável um índice que traduza o desenvolvimento sustentável. Desse modo, os selos simplificam tal análise e trazem informações objetivamente observáveis pelos consumidores(GOV1).

As opiniões dos especialistas corroboram a OECD (2008) quando enfatiza que estudos mostram que os efeitos de sustentabilidade dos esquemas de rotulagem estão crescendo com o crescente interesse dos consumidores em questões ambientais e sociais. Porém, deve-se esclarecer que estratégias de promoção de consumo sustentável focadas apenas em instrumentos informativos, tais como rótulos e selos, são ineficazes, isto porque, na prática, os consumidores estão, em grande parte, bloqueados em infraestruturas, normas sociais e hábitos que limitam severamente suas escolhas (Tukker et al., 2008; Stevens, 2010; Holt, 2012; Lorek \& Fuchs, 2013; Brizga, Mishchuk, \& Golubovska-Onisimova, 2014). Daí a importância de estabelecer relações com os demais stakeholders, principalmente governo e sociedade civil, em ações e estratégias que auxiliem a promover uma mudança cultural por meio da educação.

De acordo com os dados da pesquisa realizada pelo Ministério do Meio Ambiente (Brasil, 2012), que analisa série histórica de 2001 a 2012 sobre o que o brasileiro pensa do meio ambiente e do consumo sustentável, observa-se que os brasileiros reagem positivamente diante de um produto cujo rótulo indique fabricação ambientalmente correta. Em 2001, o percentual de respondentes que afirmaram se sentirem mais motivados diante de tal informação foi de $81 \%$, caindo para $76 \%$ em 2006 e voltando a subir em 2012, quando alcançou o equivalente a 85\%. O mesmo ocorre com relação à disposição por parte do consumidor brasileiro para comprar produtos orgânicos, que em 2001 era de $73 \%$ e em 2012 passou para 81\% (Brasil, 2012). Isto mostra que o desempenho deste papel poderá favorecer aos consumidores tomarem decisões mais racionais, em termos socioambientais, nas suas escolhas de consumo.

\subsection{PAPÉIS DAS EMPRESAS NO NÍVEL MICRO}

No nível micro também foram avaliados cinco papéis das empresas (Tabela 4). Adotar princípios de ecoeficiência foi considerado como "criticamente importante", com média geral de 4,77 e coeficiente de variação de $10 \%$ de dispersão das respostas em torno da média, nas duas rodadas, o que reforça a uniformidade das respostas dos especialistas investigados.

Tabela 4: Comparativo entre os resultados das rodadas sobre os papéis das empresas no nível micro

\begin{tabular}{|c|c|c|c|c|c|}
\hline \multirow[b]{2}{*}{ Papéis de empresas (Micro) } & \multirow{2}{*}{$\begin{array}{c}\text { Grupos de } \\
\text { especialistas }\end{array}$} & \multicolumn{2}{|c|}{$\mathbf{1}^{\mathrm{a}}$ Rodada } & \multicolumn{2}{|c|}{$2^{\mathrm{a}}$ Rodada } \\
\hline & & $\mu$ & CV & $\mu$ & $\mathbf{C V}$ \\
\hline \multirow{5}{*}{ Ecoeficiência } & Geral & 4,77 & 0,10 & 4,77 & 0,10 \\
\hline & G1 & 4,94 & 0,05 & 4,94 & 0,05 \\
\hline & $\mathrm{G} 2$ & 4,57 & 0,12 & 4,57 & 0,12 \\
\hline & G3 & 5,00 & 0,00 & 5,00 & 0,00 \\
\hline & G4 & 4,38 & 0,17 & 4,38 & 0,17 \\
\hline \multirow{5}{*}{$\begin{array}{l}\text { Analisar o ciclo de vida dos } \\
\text { produtos }\end{array}$} & Geral & 4,69 & 0,13 & 4,71 & 0,13 \\
\hline & G1 & 4,76 & 0,09 & 4,76 & 0,09 \\
\hline & G2 & 4,29 & 0,26 & 4,29 & 0,26 \\
\hline & G3 & 5,00 & 0,00 & 5,00 & 0,00 \\
\hline & G4 & 4,63 & 0,11 & 4,69 & 0,10 \\
\hline \multirow{5}{*}{ Produtos sustentáveis } & Geral & 4,85 & 0,09 & 4,85 & 0,09 \\
\hline & G1 & 4,94 & 0,05 & 4,94 & 0,05 \\
\hline & $\mathrm{G} 2$ & 4,57 & 0,17 & 4,57 & 0,17 \\
\hline & G3 & 4,86 & 0,08 & 4,86 & 0,08 \\
\hline & G4 & 4,88 & 0,07 & 4,88 & 0,07 \\
\hline Embalagens ecológicas & Geral & 4,62 & 0,14 & 4,60 & 0,14 \\
\hline
\end{tabular}

Revista de Gestão Social e Ambiental - RGSA, São Paulo, v. 12, n. 2, p. 55-70, maio/ago. 2018. 
Papéis das empresas e o consumo sustentável na visão de especialistas brasileiros

\begin{tabular}{l|l|l|l|l|l}
\hline \multirow{7}{*}{ Ecotaxas } & G1 & 4,59 & 0,16 & 4,59 & 0,16 \\
\cline { 2 - 6 } & G2 & 4,71 & 0,10 & 4,71 & 0,10 \\
\cline { 2 - 6 } & G3 & 4,71 & 0,10 & 4,71 & 0,10 \\
\hline & G4 & 4,50 & 0,17 & 4,44 & 0,16 \\
\hline & Geral & 4,18 & 0,22 & 4,15 & 0,20 \\
\hline & G1 & 4,35 & 0,18 & 4,29 & 0,18 \\
\cline { 2 - 6 } & G2 & 4,14 & 0,22 & 4,14 & 0,22 \\
\cline { 2 - 6 } & G3 & 3,86 & 0,28 & 3,86 & 0,28 \\
\cline { 2 - 6 } & G4 & 4,13 & 0,27 & 4,13 & 0,20 \\
\hline
\end{tabular}

Fonte: Pesquisa com especialistas

O conceito de ecoeficiência envolve uma combinação de eficiência ecológica com eficiência econômica, apontada no estudo de Michaelis (2003) como um novo paradigma tecnológico que envolve o comportamento das empresas. A adoção desse papel traz benefícios para as empresas que passam a ser consideradas como sustentáveis no mercado e promovem a redução dos custos e riscos de suas atividades. Os comentários adicionais de alguns especialistas ressaltam que a importância desses aspectos:

\footnotetext{
Melhorias em ecoeficiência vão além dos aspectos de conscientização ou promoção de uma cultura sustentável. Trata-se de uma estratégia de negócios. Investir em ecoeficiência significa redução de custos e riscos. E, paralelamente, pode ser revertido em vantagens competitivas aos negócios (EMP2);

Isso é ser competitivo e sustentável, produtos feitos com baixa eficiência e consumidor de recursos naturais acima do necessário, tornam estas empresas inimigas não só do meio ambiente, mas também da sociedade e de seus funcionários, pois permitem que eles sejam participantes de uma prática em desacordo com atuais metas de sustentabilidade (EMP7).
}

O World Business Council for Sustainable Development (WBCSD, 1997) defende que a melhoria da ecoeficiência é decorrente de inovações em tecnologias e práticas, o que combina as características de muitas outras abordagens e temas nas estratégias ambientais das empresas e na política do governo, tais como: "prevenção da poluição", "produção mais limpa", "análise do ciclo de vida" e "sistemas de gestão ambiental", entre outros. Neste contexto, a ecoinovação permite que as empresas integrem preocupações ambientais em um modelo de negócio convencional, com o intuito de reduzir o risco de regulação, obter a aprovação da comunidade ou a economias de custos. Porém, o especialista (ACAD5) afirma que

[...] as mudanças em direção à sustentabilidade dizem respeito às mudanças de valores, ou seja, uma revolução cultural, muito além de avanços tecnológicos, per si" (ACAD5).

Desse modo, deve-se considerar que a adoção de princípios de ecoeficiência é um elemento essencial, mas não exclusivo, em termos de sustentabilidade, uma vez que para se promover o consumo sustentável deve-se ir além de melhorias tecnológicas que focalizam a eficiência de consumo associada aos recursos utilizados nos processos produtivos.

No âmbito dessas discussões, outra questão importante na análise dos papéis das empresas é que a maior parte dos impactos ambientais dos produtos é decidida na etapa em que eles estão sendo projetados, de modo que os especialistas avaliaram a importância das empresas promoverem a análise do ciclo de vida dos produtos. As médias gerais nas duas rodadas foram de 4,69 e 4,71 e o coeficiente de variação de $13 \%$. Isto demonstra que este papel foi avaliado como "criticamente importante" pelos grupos investigados, com tendência à uniformidade nas respostas.

Ressalta-se que toda atividade econômica e os seus impactos ambientais relacionados são acionados pelo consumo, conforme afirma Tukker et al (2008). Portanto, para se promover o consumo sustentável, é necessário repensar a produção a partir dos efeitos que os produtos e o seu processo de produção causam ao meio ambiente por meio da análise do ciclo de vida dos produtos,

Revista de Gestão Social e Ambiental - RGSA, São Paulo, v. 12, n. 2, p. 55-70, maio/ago. 2018. 
desde a matéria-prima, passando pela produção até o fechamento desse o ciclo, com o uso e seu descarte. Isso inclui aspectos relacionados aos processos produtivos, recursos naturais utilizados como matéria-prima, bem como os resíduos e a poluição resultantes. Assim sendo, a relevância desse papel é ressaltada nos comentários adicionais realizados por alguns dos especialistas investigados, a saber:

A análise do ciclo de vida dos produtos vai além de um processo ecossustentável. Tem a ver com a sustentabilidade dos próprios negócios. Se meu produto esgotar sua fonte primária, fica sem ter como produzí-lo. Ou seja, é menos por 'consciência' socioambiental e mais por necessidade de encontrar formas de garantir a continuidade de seus produtos e serviços no longo prazo (EMP2);

Esta análise permite que a empresa defina quais caminhos e cuidados deverão tomar no caso de políticas de logística reversa, quando do seu produto ser normatizado para seguir esta postura, além disso, permite saber seus custos de matéria-prima, desde a sua extração até aplicação, permitindo um planejamento a longo prazo da garantia de atendimento dos atuais fornecedores" (EMP7).

Porter e Van Der Linde (1995) destacaram que o conhecimento sobre os impactos ambientais dos seus produtos e processos ainda são rudimentares em muitas empresas e indústrias, o que eleva a incerteza sobre os benefícios da inovação sustentável. Assim, a realização da análise dos ciclos de vida pode auxiliar essas empresas a entenderem que inovações em produtos e processos, com foco nos impactos ambientais, são valiosas.

Ainda no que tange às práticas internas das empresas, foi questionado aos especialistas a relevância do desenvolvimento de produtos sustentáveis para promover o consumo sustentável no contexto brasileiro, os quais também o classificaram como "criticamente importante", com médias geral de 4,85\% e coeficientes de variação de $9 \%$ de dispersão das respostas em torno da média nas duas rodadas. Segundo Tukker et al. (2008), o desenvolvimento de produtos sustentáveis pode direcionar a demanda de consumo para categorias de baixo impacto ambiental. Os comentários adicionais demonstram algumas questões que estão atreladas a esse papel, como o acesso da massa da população a tais produtos e a necessidade de ir além do "esverdeamento" dos produtos.

As empresas devem desenvolver produtos sustentáveis e, além disso, serem capazes de "massificar" a produção e o consumo desses produtos pelos consumidores, de modo que se tornem acessíveis para boa parte da população. Na medida em que os processos produtivos se tornam menos impactantes e mais "sustentáveis", há condições para que os produtos tradicionais sejam produzidos de formas mais ecoeficientes, com preços mais competitivos para os consumidores" (ACAD14);

A sustentabilidade deve alcançar não apenas o aspecto ambiental, mas também o aspecto social para que possa ser identificada como um produto sustentável. Do contrário estaremos falando de produtos verdes e não sustentáveis (ACAD16).

Uma das principais críticas associadas ao desenvolvimento de produtos sustentáveis é que, muitas vezes, o consumo sustentável é interpretado como o consumo desses produtos, sem se preocupar com o efeito rebote no aumento das suas demandas. Porém, isto não significa dizer que é inviável investir no desenvolvimento de produtos sustentáveis, mas que a capacidade de integrar as diferentes dimensões da sustentabilidade ao nível da cadeia de valor é ainda um desafio a ser superado nas discussões sobre consumo sustentável.

Além dos produtos em si, as embalagens usadas também são enfatizadas nas discussões sobre o consumo sustentável. Porter e van der Linde (1995) enfatizam que os consumidores desconhecem os custos da ineficiência de recursos nas embalagens que descartam. Elas são consideradas como um elemento essencial para a proteção dos produtos durante a sua etapa de distribuição, armazenamento, comercialização, manuseio e consumo. Porém, o seu uso causa impactos ambientais decorrentes dos recursos necessários para sua produção e dos resíduos que geram, de modo que elas são consideradas como uma das principais categorias de produtos que 
possuem apelo ecológico, conforme enfatizou Stevens (2010). Assim, os especialistas investigados foram convidados a avaliarem a relevância das empresas promoverem o uso de embalagens ecológicas. Os resultados apresentados na Tabela 4 mostram que este papel também foi classificado como "criticamente importante", com médias geral de 4,62 e 4,60, nas duas rodadas, além de 14\% de coeficientes de variação que demonstram a baixa dispersão das respostas em torno das médias.

Os comentários adicionais apresentados reforçam a importância desse papel para a promoção do consumo sustentável no contexto brasileiro. Os especialistas apontam para a necessidade de se promover a regulamentação ambiental quanto às embalagens dos produtos, bem como a relação que o uso de embalagens ecológicas pode manter com os hábitos dos consumidores.

As empresas precisam promover, mas o Estado precisa regular e definir o padrão de embalagem mais adequado para reduzir o impacto no meio ambiente (ACAD14);

Esta postura deve estar na estratégia de desenvolvimento do produto, pois é custo e não deve pesar no valor final, além disso, um custo que cai para o consumidor e não é mensurado, é o do descarte da embalagem, ficando ao cargo do poder público garantir a coleta e destinação destes, e que é cobrado do consumidor posteriormente (EMP7);

Tais práticas podem induzir os consumidores a novos hábitos, mais limpos (GOV1).

Por fim, foi avaliada a adoção de ecotaxas pelas empresas como uma forma de internalizar as externalidades ambientais dos seus produtos e processos e partilhar os custos ambientais em toda a cadeia de valor. Os resultados demonstram que este papel foi classificado como "muito importante" (Tabela 4). Mont e Peplys (2008) esclarecem que as taxas sobre os produtos são instrumentos econômicos que, embora atinjam os produtores, geralmente, são dirigidos para os consumidores. Nesse sentido, a adoção de ecotaxas levantou algumas questões entre os especialistas investigados sobre a transferência da responsabilidade dos custos ambientais dos produtos para os consumidores, o que poderia tornar a sua aquisição inviável, como se pode verificar nos comentários adicionais:

O debate sobre os custos ambientais ainda precisa avançar - tanto nas empresas, como no papel regulador do Estado e, principalmente, junto aos consumidores. Este ainda é um conceito vago e normalmente mal interpretado pelos consumidores em geral (EMP2);

Por isso, o governo precisa garantir que o cumprimento das regulamentações seja igual para todos, para que os custos ambientais não sejam apenas incorporados para aqueles que, de fato, querem promover melhorias, tornando produtos com melhor performance ambiental pouco atrativos para o consumidor final devido aos preços praticados (EMP4).

A proposta de adoção de ecotaxas deve ser utilizada, por um lado, com o propósito de inibir o consumo de produtos insustentáveis. Por outro lado, deve-se considerar que, oferecer as empresas subsídios e os incentivos fiscais, podem gerar uma relação em cadeia e influenciar o comportamento do consumidor, tornando as escolhas sustentáveis menos dispendiosas, mas que reduções de impostos incrementais ou pequenos subsídios não criam, por si só, demanda de produtos mais sustentáveis (OECD, 2008).

Com base nos dados discutidos nesta seção, pode-se considerar que o uso da técnica Policy Delphi possibilitou explorar a opinião dos especialistas sobre a relevância dos papéis que deverão ser desempenhados pelas empresas para promover o consumo sustentável no contexto brasileiro, examinando e avaliando a aceitabilidade de cada papel no geral e em cada grupo investigado.

\section{CONSIDERAÇÕES FINAIS}

Este artigo teve como objetivo analisar os papéis das empresas para promover o consumo sustentável no contexto brasileiro. Para tanto, foi realizada uma pesquisa junto aos especialistas brasileiros na temática, a partir do uso do método Policy Delphi. Analisando-se os dados obtidos 
nas duas rodadas, pôde-se inferir que os participantes da pesquisa mantiveram a tendência de relevância atribuída aos papéis de promoção do consumo sustentável obtidas na primeira rodada.

Dos papéis avaliados, apenas o lobby, para influenciar as práticas governamentais, não foi validado como relevante. $\mathrm{O}$ que parece ter mais peso para este resultado é que alguns especialistas consideram que há uma incompatibilidade entre o desempenho desse papel e os interesses econômicos das empresas. Supõe-se que essa atuação poderia ocasionar maior rigor e ampliação dos instrumentos de regulamentação ambiental, o que afetaria diretamente as atividades das empresas. Alerta-se também para o fato de que o lobby não é regulamentado no Brasil, apesar de ser muito praticado.

Assim, a principal contribuição deste estudo consiste na identificação dos papéis das empresas para promover o consumo sustentável e a sua validação junto a um conjunto de especialistas brasileiros. A maioria dos estudos relacionados a temática envolve as práticas adotadas em países europeus, de modo que os resultados discutidos podem embasar decisões sobre práticas de gestão que podem adotadas por empresas brasileiras que almejam contribuir para o desenvolvimento sustentável.

Muitos dos papéis analisados nesse estudo necessitam ainda de um processo de institucionalização para serem implantados e disseminados entre as empresas. Além disso, atenta-se para as evidências de que as condições estruturais e contextuais de promoção do consumo sustentável dependem fortemente da articulação entre as empresas, os governos e a sociedade civil, sem os quais as possibilidades de ações isoladas por parte das empresas perdem efetividade. As questões envolvidas devem ser corrigidas em ambos os lados - consumo e produção - para mitigar as externalidades socioambientais das práticas de consumo na defesa do interesse do bem público e coletivo e na promoção da sustentabilidade. Nesse contexto, as atividades de produção e de consumo fazem parte de um ciclo interdependente, em torno do qual está um conjunto de diferentes questões, iniciativas e estratégias Tukker et al., 2008; Barber, 2008; Pape, \& Heisserer, 2011; Oliveira, Correia, \& Gomez, 2016).

Apesar da evidente preocupação em evitar o desaquecimento do crescimento econômico do país, as ações de promoção do consumo sustentável devem ir além dos questionamentos sobre os aspectos econômicos, de modo a priorizar ações que melhorem a eficiência na utilização dos recursos naturais e a proteção ao meio ambiente, além de manter a qualidade de vida das pessoas. Porém, este é um dos principais aspectos relacionados ao ceticismo quanto ao envolvimento das empresas em ações e estratégias em prol da produção e do consumo sustentável, dadas ao viés economicista que, muitas vezes, prevalece como fator preponderante nos processos de tomada de decisão.

Sugere-se, portanto, desenvolver pesquisas futuras com foco em mecanismos que possibilitem a operacionalização dos papéis validados nesse estudo e avaliem seus impactos efetivos. Por meio deles, poderá ser possível monitorar os avanços da atuação das empresas, em diversos segmentos, no processo de promoção do consumo sustentável no contex to brasileiro.

\section{REFERÊNCIAS}

Abramovay, R. (2012). Muito além da economia verde. Editora Abril.

Barber, J. (2008). Mapping the movement to achieve sustainable production and consumption in North America. Journal of Cleaner Production, 15(6), 499-512.

Barbieri, J. C. (2007). Gestão ambiental empresarial: conceitos, modelos e instrumentos. In Gestão ambiental empresarial: conceitos, modelos e instrumentos. Saraiva.

Blok, V., Long, T. B., Gaziulusoy, A. I., Ciliz, N., Lozano, R., Huisingh, D., ... \& Boks, C. (2015). From best practices to bridges for a more sustainable future: advances and challenges in the transition to global sustainable production and consumption: Introduction to the Erscp stream of the Special volume. Journal of Cleaner Production, 108, 19-30.

Revista de Gestão Social e Ambiental - RGSA, São Paulo, v. 12, n. 2, p. 55-70, maio/ago. 2018. 
Bowersox, D. J., Closs, D. J., Cooper, M. B., \& Bowersox, J. C. (2014). Gestão logística da cadeia de suprimentos. Porto Alegre: Bookman.

Brasil (2012). O que o brasileiro pensa do meio ambiente e do consumo sustentável: Brasília: MMA.

Brito, R. P., \& Berardi, P. C. (2010). Vantagem competitiva na gestão da cadeia de suprimentos: um metaestudo. In: RAE. São Paulo, 50(2), 155-169.

Brizga, J., Mishchuk, Z., \& Golubovska-Onisimova, A. (2014). Sustainable consumption and production governance in countries in transition. Journal of cleaner production, 63, 45-53.

Carroll, A. B. (1998). The four faces of corporate citizenship. Business and society review, 100(1), 1-7.

Clark, G. (2007). Evolution of the global sustainable consumption and production policy and the United Nations Environment Programme's (Unep) supporting activities. Journal of cleaner production, 15(6), 492498.

Consumers International; MMA; MEC/IDEC (2005). Consumo sustentável: manual de Educação. Brasília: Consumers International, MMA, MEC/Idec.

Costa, D. V., \& Teodósio, A. D. S. D. S. (2011). Desenvolvimento sustentável, consumo e cidadania: um estudo sobre a (des) articulação da comunicação de organizações da sociedade civil, do estado e das empresas. Revista de Administração Mackenzie, 12(3), 114-145.

Franklin, K. K., \& Hart, J. K. (2007). Idea generation and exploration: benefits and limitations of the policy Delphi research method. Innovative Higher Education, 31(4), 237-246.

Fuchs, D. A., \& Lorek, S. (2005). Sustainable consumption governance: A history of promises and failures. Journal of Consumer Policy, 28(3), 261-288.

Holt, D. B. (2012). Constructing sustainable consumption: From ethical values to the cultural transformation of unsustainable markets. The Annals of the American Academy of Political and Social Science, 644(1), 236255.

Jackson, T. (2006). Sustainable consumption. Handbook of sustainable development. Edited by Giles Atkinson, Simon Dietz, Eric Neumayer.

Jackson, T., \& Michaelis, L. (2003). Policies for sustainable consumption. Sustainable Development Commission, London.

Lim, W. M. (2017). Inside the sustainable consumption theoretical toolbox: critical concepts for sustainability, consumption, and marketing. Journal of Business Research, 78, 69-80.

Liu, W., Oosterveer, P., \& Spaargaren, G. (2016). Promoting sustainable consumption in China: A conceptual framework and research review. Journal of Cleaner Production, 134, 13-21.

Lorek, S., \& Fuchs, D. (2013). Strong sustainable consumption governance-precondition for a degrowth path? Journal of cleaner production, 38, 36-43.

Lorek, S., \& Spangenberg, J. H. (2014). Sustainable consumption within a sustainable economy-beyond green growth and green economies. Journal of cleaner production, 63, 33-44.

Michaelis, L. (2003). The role of business in sustainable consumption. Journal of Cleaner Production, 11(8), 915-921.

Mont, O., \& Plepys, A. (2008). Sustainable consumption progress: should we be proud or alarmed? Journal of Cleaner Production, 16(4), 531-537.

Muster, V. (2011). Companies promoting sustainable consumption of employees. Journal of consumer policy, 34(1), 161-174.

OECD (2008). Promoting Sustainable Consumption. Good practices in OECD countries. OECD Publications: Paris, France.

Oliveira, J. A. P. (2013). Empresas na sociedade: sustentabilidade e responsabilidade social. Elsevier Editora. 
Oliveira, V. M., Correia, S. É. N., \& Gomez, C. R. P. (2016). Cultura de consumo, sustentabilidade e práticas empresariais: como as empresas podem contribuir para promover o valor simbólico da sustentabilidade nas atividades de consumo? Revista de Gestão Ambiental e Sustentabilidade, 5(1), 61.

Pape, J., \& Heisserer, B. (2011). Towards sustainable consumption: a conceptualisation and evaluation of measurement tools.

Peattie, K., (2007). Toward sustainable organizations for the 21st century. 21st Century management: a reference handbook. Sage Publications.

Málovics, G., Csigéné, N. N., \& Kraus, S., (2008). The role of corporate social responsibility in strong sustainability. The Journal of Socio-Economics, 37, 907-918.

Pires, S. R. (2009). Gestão da cadeia de suprimentos: conceitos, estratégias, práticas e casos. São Paulo: Atlas.

Porter, M. E., \& Kramer, M. R. (2011). Creating shared value: how to reinvent capitalism—And unleash a wave of innovation and growth. Harvard Business Review, 2-17.

Porter, M. E., \& Van der Linde, C. (1995). Toward a new conception of the environment-competitiveness relationship. The Journal of Economic Perspectives, 9(4), 97-118.

Portilho, F. (2005). Sustentabilidade ambiental, consumo e cidadania. Cortez.

Rattis Teixeira, P. M (2011). Empreendimentos em consumo sustentável: um estudo da atuação pública e política da sociedade civil organizada. Dissertação (Mestrado). Universidade Federal de São Carlos Ciência, Tecnologia e Sociedade.

Rayens, M. K., \& Hahn, E. J. (2000). Building consensus using the policy Delphi method. Policy, politics, \& nursing practice, 1(4), 308-315.

Schrader, U., \& Thøgersen, J. (2011). Putting sustainable consumption into practice. Journal of Consumer Policy, 34(1), 3-8.

Shao, J., Taisch, M., \& Mier, M. O. (2017). Influencing factors to facilitate sustainable consumption: from the experts' viewpoints. Journal of Cleaner Production, 142, 203-216.

Silva, M. E., \& Cândido, G. C. (2014). The business contribution for sustainable consumption: A proposal of theoretical categories and analytical parameters. Revista Eletrônica de Ciência Administrativa, 13(1), 74.

Stevens, C. (2010). Linking sustainable consumption and production: the government role. In Natural Resources Forum, 34(1), 16-23. Blackwell Publishing Ltd.

Tukker, A., Emmert, S., Charter, M., Vezzoli, C., Sto, E., Andersen, M. M., \& Lahlou, S. (2008). Fostering change to sustainable consumption and production: an evidence based view. Journal of cleaner production, 16(11), 1218-1225.

Turoff, M. (1997). Alternative futures for distance learning: The force and the darkside. Virtual Learning Environments and the Role of the Teacher.

WBCSD (1997). Eco-efficiency: the business link to sustainable development. Cambridge, MA: MIT Press.

Data da submissão: 17/09/2017

Data de aceite: 07/12/2017 